\title{
Diagnóstico de qualidade ambiental do Parque Estadual Vitório Piassa por meio do uso de besouros bioindicadores
}

\author{
Aline Bugoni ${ }^{1 *}$ \\ Malva Isabel Medina Hernández ${ }^{2}$ \\ Rodrigo Lingnau ${ }^{3}$ \\ ${ }^{1}$ Programa de Pós-Graduação em Desenvolvimento Regional \\ Universidade Tecnológica Federal do Paraná, Via do Conhecimento, Km 1 \\ CEP 85503-390, Pato Branco - PR, Brasil \\ ${ }^{2}$ Laboratório de Ecologia Terrestre Animal, Departamento de Ecologia e Zoologia \\ Universidade Federal de Santa Catarina, Florianópolis - SC, Brasil \\ ${ }^{3}$ Universidade Tecnológica Federal do Paraná, Francisco Beltrão - PR, Brasil \\ * Autor para correspondência \\ aline.bug@hotmail.com
}

Submetido em 26/09/2016

Aceito para publicação em 08/06/2017

\section{Resumo}

O presente trabalho teve como objetivo analisar a qualidade ambiental em um fragmento florestal de Mata Atlântica próximo a uma área urbana. O estudo foi realizado no Parque Estadual Vitório Piassa, situado no município de Pato Branco, Paraná, onde coletas de besouros da subfamília Scarabaeinae foram realizadas através de armadilhas de queda em três áreas distintas dentro do parque, entre fevereiro e março de 2015 . Foram coletados no total 945 indivíduos pertencentes a 22 espécies, dos quais 366 indivíduos de 18 espécies foram coletados na área de vegetação mais densa, 291 de 18 espécies na área de vegetação secundária e 288 de 16 espécies na área mais antropizada. As espécies mais abundantes foram Dichotomius mormon e Eurysternus parallelus, somando juntas mais de 50\% do total de indivíduos capturados. Os índices de diversidade (Shannon e Simpson) e de equitabilidade (Pielou) foram maiores na área com vegetação secundária, o que pode ser explicado por ser uma área intermediária, tanto em termos espaciais como temporais. A análise de similaridade baseada no índice de Bray-Curtis mostrou a formação de dois grupos: um constituído pelas assembleias das áreas em regeneração e o outro apenas pela área de vegetação mais densa, principalmente devido à grande abundância de besouros nesta área. Assim, foi possível observar que o Parque mantém uma qualidade de habitat que permite abrigar uma grande abundância de muitas espécies de escarabeíneos, principalmente nas áreas mais conservadas.

Palavras-chave: Coleoptera; Fragmentação; Mata Atlântica; Parque urbano; Scarabaeinae

\section{Abstract}

Evaluation of environmental quality of Vitório Piassa State Park through the use of bioindicator beetles. The objective of the present study was to analyze the environmental quality of a forest fragment of the Atlantic Forest close to an urban area. The study was carried out in Vitório Piassa State Park, located in the municipality of Pato Branco, Paraná, Brazil. Beetles from the subfamily Scarabaeinae were collected with pitfall traps in three different areas in the park, between February and March 2015. A total of 945 individuals and 22 
species were collected, of which 366 individuals and 18 species were found in the densest vegetation area, 291 individuals and 18 species in the secondary vegetation area, and 288 individuals and 16 species in the most anthropized area. The most abundant species were Dichotomius mormon and Eurysternus parallelus, accounting for more than $50 \%$ of the total captured individuals. Diversity indices (Shannon and Simpson) and equitability (Pielou) were higher in the area with secondary vegetation, probably because it is an intermediate area, both in spatial and temporal terms. Similarity analysis based on the Bray-Curtis index showed the formation of two groups: one consisting of the assemblages of regenerating areas and the other only of the densest vegetation area, mainly due to the great abundance of beetles in this area. Thus, it was possible to observe that the Park maintains a habitat quality that allows the sheltering of a great abundance of many dung beetle species, mainly in the most conserved areas.

Key words: Atlantic Forest; Coleoptera; Fragmentation; Scarabaeinae; Urban park

\section{Introdução}

O bioma Mata Atlântica possui uma grande diversidade de espécies e está entre as regiões ameaçadas do planeta, sendo uma das áreas prioritárias para conservação no planeta (MYERS et al., 2000). Atualmente se encontra sob forte pressão antrópica, o que explica a extinção de diversas espécies ao longo dos anos (TABARELLI et al., 2005; SOS MATA ATLÂNTICA, 2014). O processo de desmatamento e fragmentação de habitat é considerado uma das principais causas de perda de espécies em comunidades biológicas (PIRES et al., 2006) e, por esse motivo, vem sendo apontado como "uma das maiores ameaças à biodiversidade" (PAGLIA et al., 2006, p. 281). Esse problema tem como causa primordial a intervenção humana crescente nos ambientes naturais (CARVALHO et al., 2004; OLIFIERS; CERQUEIRA, 2006). Outra consequência da fragmentação é o efeito de borda (PIRES et al., 2006), pelo fato de se formarem fragmentos separados uns dos outros, ocorrem diferenças no microclima do interior e da borda do fragmento, sendo que essas mudanças podem ser notadas muitos metros para dentro do fragmento. $\mathrm{O}$ efeito de borda se dá pelo aumento da luminosidade, da temperatura (tanto do solo quanto do ar), pela diminuição da umidade e maior incidência de ventos que adentram os fragmentos florestais (PRIMACK; RODRIGUES, 2001; OLIFIERS; CERQUEIRA, 2006). O fato de algumas espécies serem adaptadas a certa temperatura ou luminosidade faz com que muitas espécies se extingam, por não se adaptarem ao novo ambiente formado, por conta das mudanças desses fatores no fragmento. A característica de algumas espécies evitarem a borda torna-as ainda mais suscetíveis à extinção (PRIMACK; RODRIGUES, 2001; PIRES et al., 2006).

O desmatamento e a fragmentação de habitat também ameaçam as espécies em longo prazo já que a perda de habitat e o isolamento dos fragmentos diminuem o fluxo de genes, culminando na extinção de espécies. A fragmentação diminui a capacidade de disseminação e povoação de algumas espécies, uma vez que o risco de predação faz com que estas evitem atravessar até mesmo pequenas faixas desprovidas de vegetação, o que explica o fato de algumas áreas não serem recolonizadas após a extinção de sua população original. Consequentemente, a dispersão de sementes também será afetada, ocasionando a extinção de plantas com frutos ou sementes. Por conseguinte, animais que se alimentam desses frutos também passarão a sofrer pela falta de alimento, ocasionando um "efeito em cascata" dentro dos ecossistemas e a extinção de uma espécie leva à extinção de outra (PRIMACK; RODRIGUES, 2001; SOFIA; SUZUKI, 2004).

Uma das medidas que visam diminuir os impactos causados pelo desmatamento e a fragmentação florestal, além de conservar os ambientes naturais, é a criação de Unidades de Conservação (UCs). As UCs, de acordo com a forma de manejo, contribuem para o sistema de suporte de vida silvestre, para a preservação da diversidade genética e para a utilização sustentável dos recursos naturais, para a recreação e o turismo ecológico. Essas áreas podem, ainda, promover a ligação entre os ecossistemas naturais por meio de corredores ecológicos, criando uma rede de áreas conservadas em vez de um mosaico de fragmentos e matrizes, diminuindo 
assim os efeitos da fragmentação (MORSELLO, 2001; MMA, 2014). No século XX, essa medida se propagou efetivamente devido à alta taxa de extinção de espécies (BENSUSAN, 2006) e em 1988 a Constituição Federal do Brasil, em seu art. 225, assegurou a todos um "meio ambiente ecologicamente equilibrado" e determinou ao poder público "o dever de defendê-lo e preservá-lo para as presentes e futuras gerações" (BENSUSAN, 2006). A partir da Constituição Federal, foi criado um Sistema Nacional de Unidades de Conservação - SNUC e, após dez anos de elaboração e negociações, resultou na Lei no 9.985 de 2000 (SNUC, 2000), a qual estabelece as funcionalidades de cada ambiente concebido. Essas unidades, de acordo com o SNUC, se dividem em dois grupos: Unidades de Proteção Integral, que permitem apenas o uso indireto dos seus recursos naturais em ações voltadas à pesquisa científica e turismo ecológico, e Unidades de Uso Sustentável, que têm como objetivo principal compatibilizar a conservação da natureza com o uso sustentável de seus recursos naturais (SNUC, 2006), estando inserida no grupo de Proteção Integral a categoria "Parque". No entanto, muitas normas e leis específicas de cada categoria de Unidade de Conservação não são cumpridas e mesmo que os números de UCs criadas no Brasil sejam altos, é preciso de fato proteger essas áreas, já que prevalecem muitas ameaças que podem levar à extinção de espécies (WWF BRASIL, 2014).

A manutenção da biodiversidade depende da conservação dos ecossistemas. Quando as características básicas de um ecossistema são alteradas, muitas das espécies presentes nesse ambiente não serão capazes de se adaptarem às novas condições ambientais impostas, deixando de integrar esse ecossistema, alterando toda a cadeia alimentar necessária para a sobrevivência das espécies (WINK et al., 2005; LOPES et al., 2011; SILVA et al., 2014). Nas florestas tropicais, a extinção de espécies é relativamente alta, principalmente pelo fato de as espécies serem representadas por um número pequeno de indivíduos e por participarem de interações ecológicas restritas e complexas com outras espécies (THOMAZINI; THOMAZINI, 2000; BOGONI et al., 2016). A extinção de algumas espécies de vertebrados em fragmentos florestais ocasiona um efeito em cascata nos ecossistemas, interferindo em outros grupos de animais, bem como nos processos ecológicos que esses indivíduos desempenham como a dispersão secundária de sementes e a decomposição de excrementos (KLEIN, 1989). Assim, os efeitos dos impactos ambientais causados pela intervenção humana nos ecossistemas, podem ser observados através da análise dos indivíduos presentes nesses ambientes (THOMAZINI; THOMAZINI, 2000; FREITAS et al., 2006; AUDINO et al., 2007). Esses indivíduos são chamados indicadores biológicos ou bioindicadores, pois permitem avaliar alguns aspectos estruturais dos ecossistemas, através de alterações na abundância, na diversidade e na composição do grupo de indicadores, medindo assim a perturbação do ambiente, sendo essencial que esses indicadores biológicos sejam indivíduos sensíveis aos efeitos das alterações nos ecossistemas (BROWN, 1997). Os insetos, por serem sensíveis a quase todos os tipos de alterações no ambiente, são considerados os melhores indicadores de alterações em habitat ou em comunidades que habitam um mesmo ecossistema (FREITAS et al., 2006). Os coleópteros têm sido amplamente utilizados (THOMAZINI; THOMAZINI, 2000; WINK et al., 2005) devido à facilidade de amostragem, alta diversidade de espécies nas florestas tropicais e por serem sensíveis aos impactos do desmatamento (KLEIN, 1989; HALFFTER, 1991). Os coleópteros da subfamília Scarabaeinae estão entre as espécies utilizadas como bioindicadores pois a degradação do habitat leva à redução da riqueza, da abundância e da biomassa dos escarabeíneos (GARDNER et al., 2008). Portanto, com a finalidade de observar a qualidade ambiental em um fragmento de floresta tropical remanescente de Mata Atlântica, dentro de um centro urbano no município de Pato Branco, Paraná, Brasil, foram utilizados besouros da subfamília Scarabaeinae com o objetivo de identificar áreas mais biodiversas dentro da extensão total do parque.

\section{Material e Métodos}

\section{Área de estudo}

O estudo foi realizado no Parque Estadual Vitório Piassa (PEVP), localizado no município de Pato Branco, na região sudoeste do estado do Paraná, Brasil (26 $6^{\circ} 11^{\prime} 42^{\prime \prime}$ S e $\left.52^{\circ} 40^{\prime} 49^{\prime \prime} \mathrm{W}\right)$, estando a uma altitude 
de $760 \mathrm{~m}$. O PEVP está situado às margens da via do Conhecimento (PR-493) e da Universidade Tecnológica Federal do Paraná (UTFPR), em proximidade ao centro urbano da cidade de Pato Branco (Figura 1). Abrange uma área de 107,23 ha de remanescente de Mata Atlântica, caracterizada como Floresta Ombrófila Mista (RODERJAN et al., 2002), coberta por espécies nativas, como Araucária (Araucaria angustifolia (Bertol.) Kuntze, 1898) e Peroba-rosa (Aspidosperma polyneuron (Mueller Argoviensis) Mart., 1860) e algumas espécies exóticas (ex. Eucaliptus spp. e Pinnus spp.). A área foi utilizada para extração e beneficiamento de madeira com uma serraria em atividade até o ano de 1983 (VOLTOLINI, 2000). O local foi instituído como Parque Estadual, através do Decreto 5169 de 30 de julho de 2009, após negociações com a Prefeitura Municipal juntamente com o Instituto Ambiental do Paraná (IAP).

\section{Coleta de besouros indicadores}

Para a captura dos bioindicadores de qualidade ambiental no PEVP, foram feitas duas campanhas de coletas de coleópteros da subfamília Scarabaeinae nos meses de fevereiro e março de 2015. A primeira coleta ocorreu no período de 22 a 24 de fevereiro de 2015 e a segunda coleta entre os dias 18 a 20 de março de 2015, sempre respeitando o prazo de $48 \mathrm{~h}$ entre a colocação das armadilhas e o recolhimento dos indivíduos capturados em cada uma delas. As coletas foram realizadas com licença de coleta ICMBio/SISBio

FIGURA 1: Mapa do Parque Estadual Vitório Piassa, Pato Branco - PR, Brasil, assinalando as três áreas de coleta de besouros escarabeíneos.

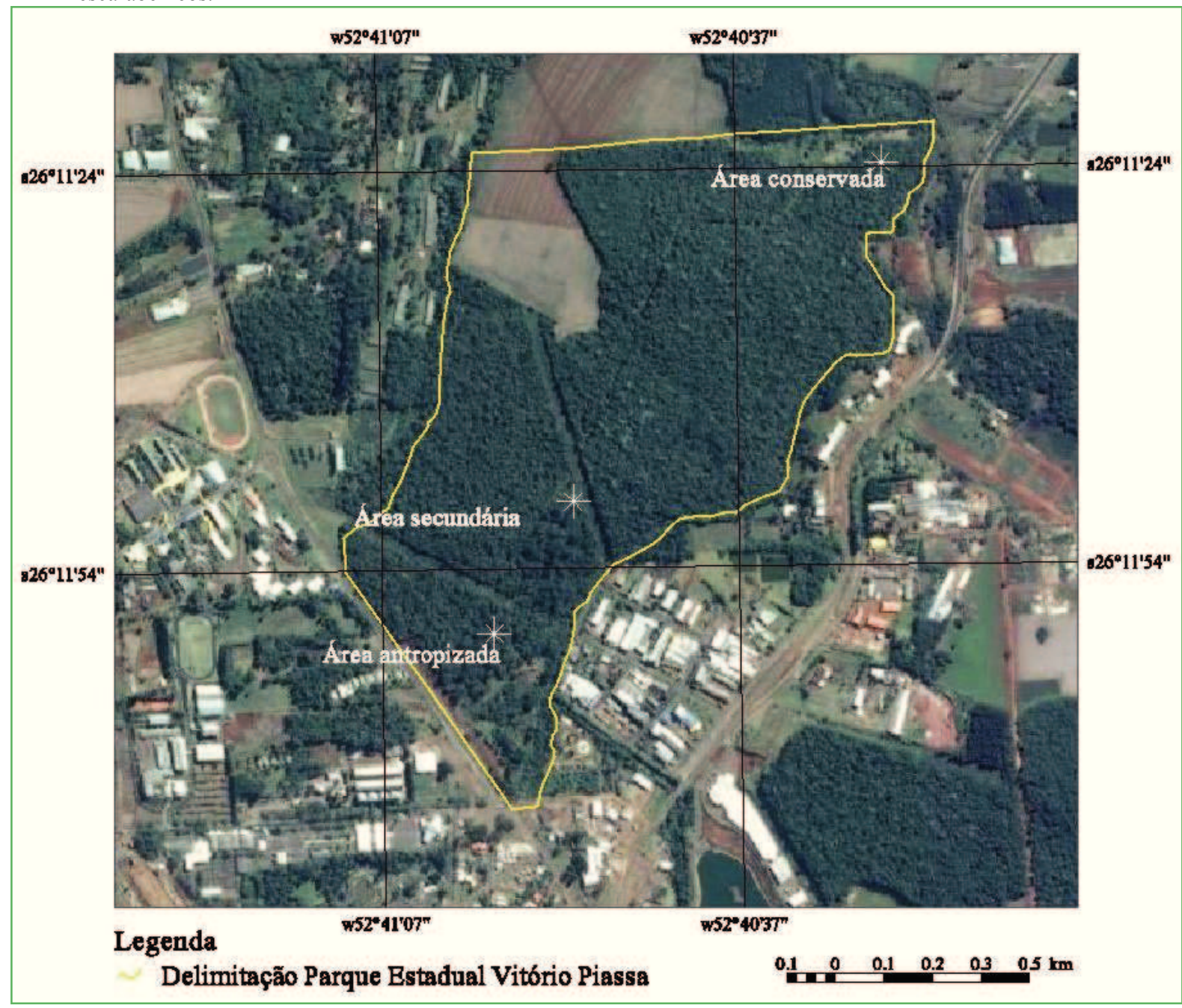


44127-1 e com autorização de permanência no Parque, fornecida pelo Instituto Ambiental do Paraná (IAP). Foram estabelecidos três sítios amostrais no fragmento, de acordo com três classes de alteração ambiental (Figura 1): o primeiro sítio de coleta foi realizado em uma área aparentemente conservada, com vegetação mais densa, nomeada na pesquisa como Área Conservada (Figura 1). O segundo sítio situou-se mais ao centro do parque, constituído de vegetação secundária, área com maior incidência solar, árvores de troncos mais finos, cipós e predominância de gramíneas em seu solo, nomeado na pesquisa como Área Secundária (Figura 1). O terceiro sítio de coleta é uma área bastante antropizada, localizado próximo à região de tratamento de esgotos da cidade e à rodovia PR-493, local de solo compacto e presença de alguns resíduos de construções, sendo nomeado como Área Antropizada (Figura 1). A distância entre os sítios 1 e 2 é de $1 \mathrm{~km}$ e do sítio 2 ao sítio 3 foi de mais de $300 \mathrm{~m}$.

Em cada um dos três locais foram colocadas 10 armadilhas para a captura dos besouros, totalizando assim um esforço amostral de 60 armadilhas. Foram demarcados cinco pontos de amostragem ao longo de cada trilha e em cada ponto foram fixadas duas armadilhas de queda, do tipo pitfall, com $10 \mathrm{~m}$ de distância entre si, uma para captura de espécies necrófagas e outra para espécies coprófagas. As armadilhas foram confeccionadas utilizando-se potes plásticos com $20 \mathrm{~cm}$ de diâmetro e $10 \mathrm{~cm}$ de altura, que foram enterrados no solo até a linha da borda e protegidos por uma tampa de plástico com furo central, onde foi suspensa a isca de atração, de acordo com seu hábito alimentar. Para atrair as espécies necrófagas foram utilizados aproximadamente $10 \mathrm{~g}$ de iscas, sendo estas compostas de pedaços de carne suína em decomposição, e para as coprófagas foram utilizadas $10 \mathrm{~g}$ de fezes humanas. No interior das armadilhas foi adicionada uma mistura de água e $2 \%$ de detergente líquido neutro, após $48 \mathrm{~h}$ de exposição, os insetos capturados em cada armadilha foram separados e colocados em recipientes (Falcons) individuais, identificados com os dados de cada coleta (local de coleta, mês, tipo de isca e número do pitfall) e conservados em álcool $70 \%$ para posterior triagem das espécies em laboratório.

\section{Análise de dados}

Os besouros escarabeíneos coletados foram separados, montados e identificados a nível específico. O material foi enviado para o Laboratório de Ecologia Terrestre Animal (LECOTA) da Universidade Federal de Santa Catarina (UFSC) para a identificação destas espécies de acordo com a coleção de referência do mesmo laboratório. Algumas espécies não identificadas foram enviadas para o taxonomista Dr. Fernando Zagury Vaz-de-Mello da Universidade Federal do Mato Grosso (UFMT). Foi calculada a riqueza de espécies e a abundância de indivíduos em cada sítio de coleta e feito um gráfico de distribuição de abundância para cada área. Posteriormente foram calculados os índices de diversidade de espécies, Simpson e Shannon-Wiener, e também o índice de equitabilidade de Pielou, que indica como está distribuída a abundância total pelas espécies de cada assembleia. Foi definida a suficiência amostral comparando o número de espécies amostrado com o número de espécies estimado por Chao $1 \mathrm{e}$ foi comparada a riqueza de espécies entre os sítios, utilizando o programa EstimateS v. 9.1.0 (COLWELL, 2005). Para calcular a similaridade entre as áreas, foi utilizado o índice de Bray-Curtis e foram construídos dendrogramas pelo método do encadeamento médio para uma melhor visualização dos resultados encontrados. Essas análises foram realizadas no programa PAST 3.1 (PAleontological STatistics, HAMMER et al., 2001).

\section{Resultados}

Foram coletados 945 indivíduos da subfamília Scarabaeinae no PEVP, que se dividem taxonomicamente em 22 espécies pertencentes a nove gêneros (Tabela 1). Destas, apenas duas espécies não foram identificadas a nível específico. As quatro espécies mais abundantes em todas as áreas foram: Dichotomius mormon (Ljungh,1799), com 275 indivíduos (29,1\% do total de indivíduos coletados), Eurysternus parallelus Castelnau, 1840, com 213 indivíduos (22,5\% do total), Dichotomius sericeus (Harold, 1867), com 165 indivíduos (17,5\%) e Onthophagus catharinensis Paulian, 1936, com 132 indivíduos (14\%) (Figura 2). 
TABELA 1: Besouros copro-necrófagos da subfamília Scarabaeinae coletados em Pato Branco - PR e abundância de indivíduos em três ambientes dentro do Parque Estadual Vitório Piassa nos meses de fevereiro e março de 2015.

\begin{tabular}{|c|c|c|c|c|}
\hline \multirow{2}{*}{ Espécie } & \multicolumn{3}{|c|}{ Área } & \multirow{2}{*}{ Tota } \\
\hline & Conservada & Secundária & Antropizada & \\
\hline Canthidium aff. trinodosum & 5 & 5 & 5 & 15 \\
\hline Canthidium lucidum & 0 & 0 & 1 & 1 \\
\hline Canthon angularis & 2 & 7 & 3 & 12 \\
\hline Canthon auricollis & 1 & 0 & 0 & 1 \\
\hline Canthon lividus seminitens & 0 & 1 & 3 & 4 \\
\hline Canthon ibarragrassoi & 6 & 6 & 3 & 15 \\
\hline Coprophanaeus saphirinus & 2 & 1 & 0 & 3 \\
\hline Deltochilum brasiliense & 10 & 6 & 5 & 21 \\
\hline Deltochilum morbillosum & 4 & 1 & 0 & 5 \\
\hline Deltochilum rubripenne & 4 & 2 & 1 & 7 \\
\hline Dichotomius assifer & 6 & 8 & 1 & 15 \\
\hline Dichotomius mormon & 148 & 65 & 62 & 275 \\
\hline Dichotomius sericeus & 35 & 69 & 61 & 165 \\
\hline Dichotomius sp. & 1 & 0 & 0 & 1 \\
\hline Eurysternus caribaeus & 0 & 1 & 0 & 1 \\
\hline Eurysternus cyanescens & 8 & 8 & 2 & 18 \\
\hline Eurysternus parallelus & 68 & 39 & 106 & 213 \\
\hline Ontherus azteca & 15 & 9 & 7 & 31 \\
\hline Ontherus sulcator & 0 & 0 & 1 & 1 \\
\hline Onthophagus catharinensis & 48 & 58 & 26 & 132 \\
\hline Onthophagus tristis & 1 & 1 & 0 & 2 \\
\hline Scybalocanthon nigriceps & 2 & 4 & 1 & 7 \\
\hline Total de indivíduos & 366 & 291 & 288 & 945 \\
\hline Total de espécies & 18 & 18 & 16 & 22 \\
\hline Índice de diversidade Shannon & 1,90 & 2,08 & 1,75 & \\
\hline Índice de diversidade Simpson & $\mathbf{0 , 7 7}$ & $\mathbf{0 , 8 3}$ & 0,76 & \\
\hline Índice de equitabilidade Pielou & 0,66 & 0,72 & 0,63 & \\
\hline
\end{tabular}

As armadilhas para espécies coprófagas atraíram 856 indivíduos, totalizando $90,6 \%$ do total coletado. Já as armadilhas para necrófagos capturaram 89 indivíduos, representando apenas $9,4 \%$ dos insetos coletados. As 11 espécies coletadas que apresentaram uma abundância mínima maior que 10 indivíduos foram classificadas de acordo com seu hábito alimentar. Desta forma, as espécies preferencialmente coprófagas foram: Canthidium aff. trinodosum (Bohenann, 1858), Canthon angularis, Canthon ibarragrassoi Martinez, 1952, Deltochilum brasiliense (Castelnau, 1840), Dichotomius assifer (Eschscholtz, 1822), Dichotomius mormon, Eurysternus parallelus Castelnau, 1840 e Onthophagus catharinensis Paulian, 1936. As espécies generalistas foram: Dichotomius sericeus (Harold,
1867), Eurysternus cyanescens Balthasar, 1939 e Ontherus azteca Harold, 1869. Não foram encontradas espécies exclusivamente necrófagas.

\section{Comparação entre áreas de coleta}

As coletas realizadas nas três áreas do parque mostraram um grande número de espécies generalistas em relação ao habitat, encontrando-se nas três áreas de coleta as espécies Canthidium aff. trinodosum (Bohenan, 1858), Canthon angularis Harold, 1868, Canthon ibarragrassoi Martinez, 1952, Deltochilum brasiliense (Castelnau, 1840), Deltochilum rubripenne (Gory, 1831), Dichotomius assifer (Eschscholtz, 1822), Dichotomius 
FIGURA 2: Diagramas de distribuição de abundância das espécies de escarabeíneos coletadas em três áreas dentro do Parque Estadual Vitório Passa, Pato Branco - PR, Brasil, no verão de 2015.

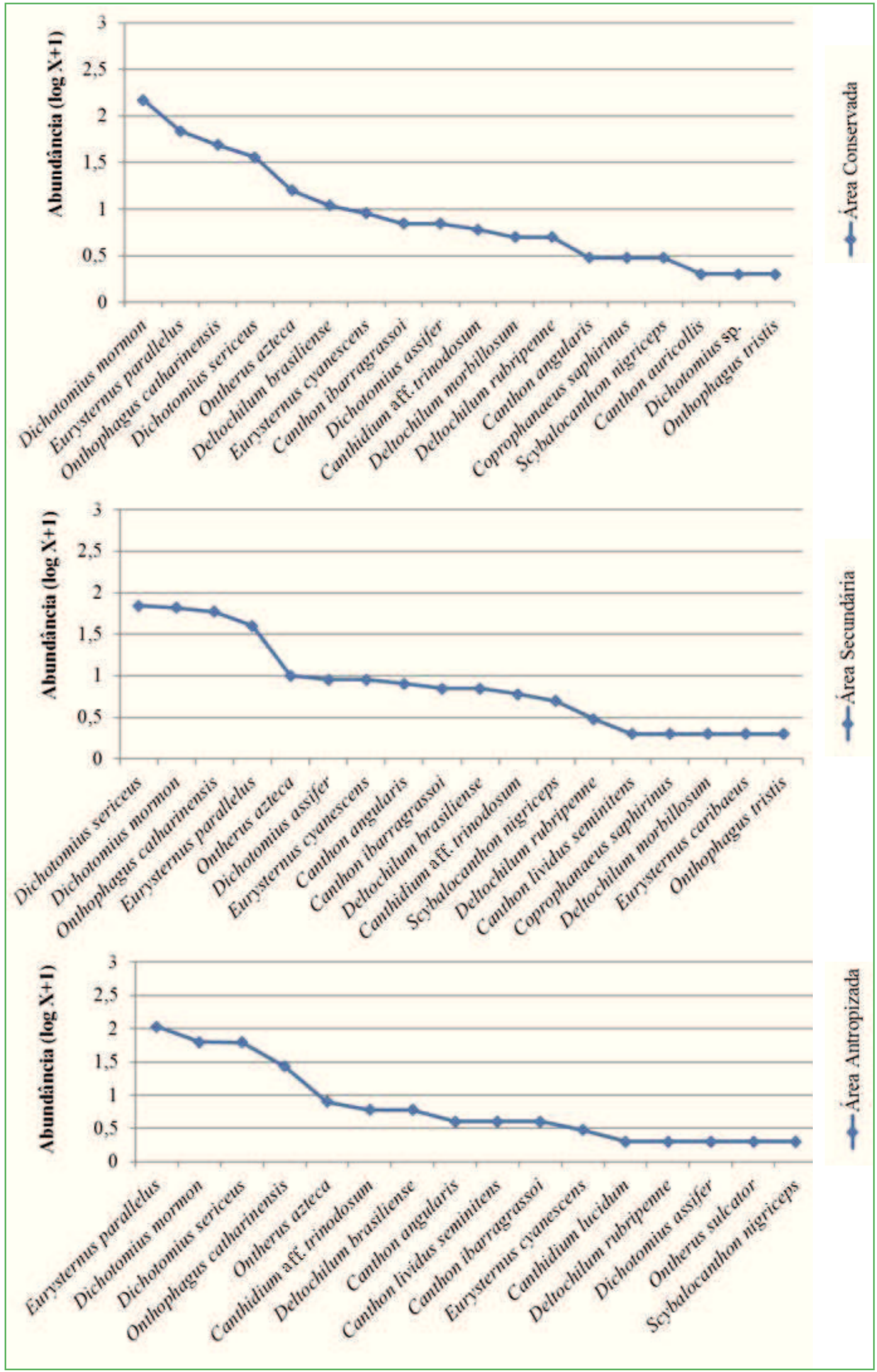


mormon (Ljungh,1799), Dichotomius sericeus (Harold, 1867), Eurysternus cyanescens Balthasar, 1939, Eurysternus parallelus Castelnau, 1840, Ontherus azteca Harold, 1869, Onthophagus catharinensis Paulian, 1936, Scybalocanthon nigriceps (Harold, 1868). Já as espécies Canthon auricollis Redtenbacher, 1867 e Dichotomius sp. foram encontrados apenas no local de coleta denominado Área Conservada. A espécie Eurysternus caribaeus (Herbst, 1789) foi encontrada somente na Área Secundária. Canthidium lucidum Harold, 1867 e Ontherus sulcator (Fabricius, 1775) foram encontrados especificamente na Área Antropizada, entretanto, Coprophanaeus saphirinus (Sturm, 1826), Deltochilum morbillosum Burmeister, 1848 e Onthophagus tristis Harold, 1873, foram coletados em ambas as áreas com vegetação densa e vegetação secundária, e uma única espécie, Canthon lividus seminitens Harold, 1868, foi encontrada exclusivamente nas áreas conservada e antropizada (Tabela 1).

As medidas ecológicas das comunidades provenientes das três áreas de coleta estão expostas na Tabela 1, onde se pode observar que a abundância da Área Conservada foi maior, apresentando 366 indivíduos.
A riqueza de espécies foi igual na Área Conservada e Área Secundária (18 espécies), entretanto, a área com vegetação secundária apresentou um número menor de indivíduos (291 indivíduos coletados), com apenas uma espécie restrita a esta área, diferenciando-se da Área Conservada, que teve duas espécies exclusivas desse sítio. Os índices de diversidade (Shannon e Simpson) e de equitabilidade (Pielou) foram um pouco maiores na Área Secundária e menores na Área Antropizada.

As curvas de acumulação de espécies, efetuadas a partir da riqueza acumulada de espécies em cada área de coleta, mostraram uma boa suficiência amostral, já que se nota que as curvas tendem a se estabilizar, indicando que as coletas conseguiram capturar a maioria das espécies existentes no PEVP (Figura 3). O número de espécies estimadas pelo método Chao 1 foi 18,74 espécies para a Área Conservada, ou seja, foi possível capturar $96 \%$ das espécies que devem existir no local; da mesma forma, na Área Secundária o cálculo foi de 22,98 espécies, ou seja, houve uma suficiência de 78\%; na Área Antropizada o estimador indicou 20,98 espécies e assim as 16 espécies representam $76 \%$ do estimado.

FIGURA 3: Curvas de acumulação de espécies calculadas para as assembleias de Scarabaeinae do Parque Estadual Vitório Piassa, coletados nos meses de fevereiro e março de 2015. Área Conservada (Área 1); Área Secundária (Área 2); Área Antropizada (Área 3).

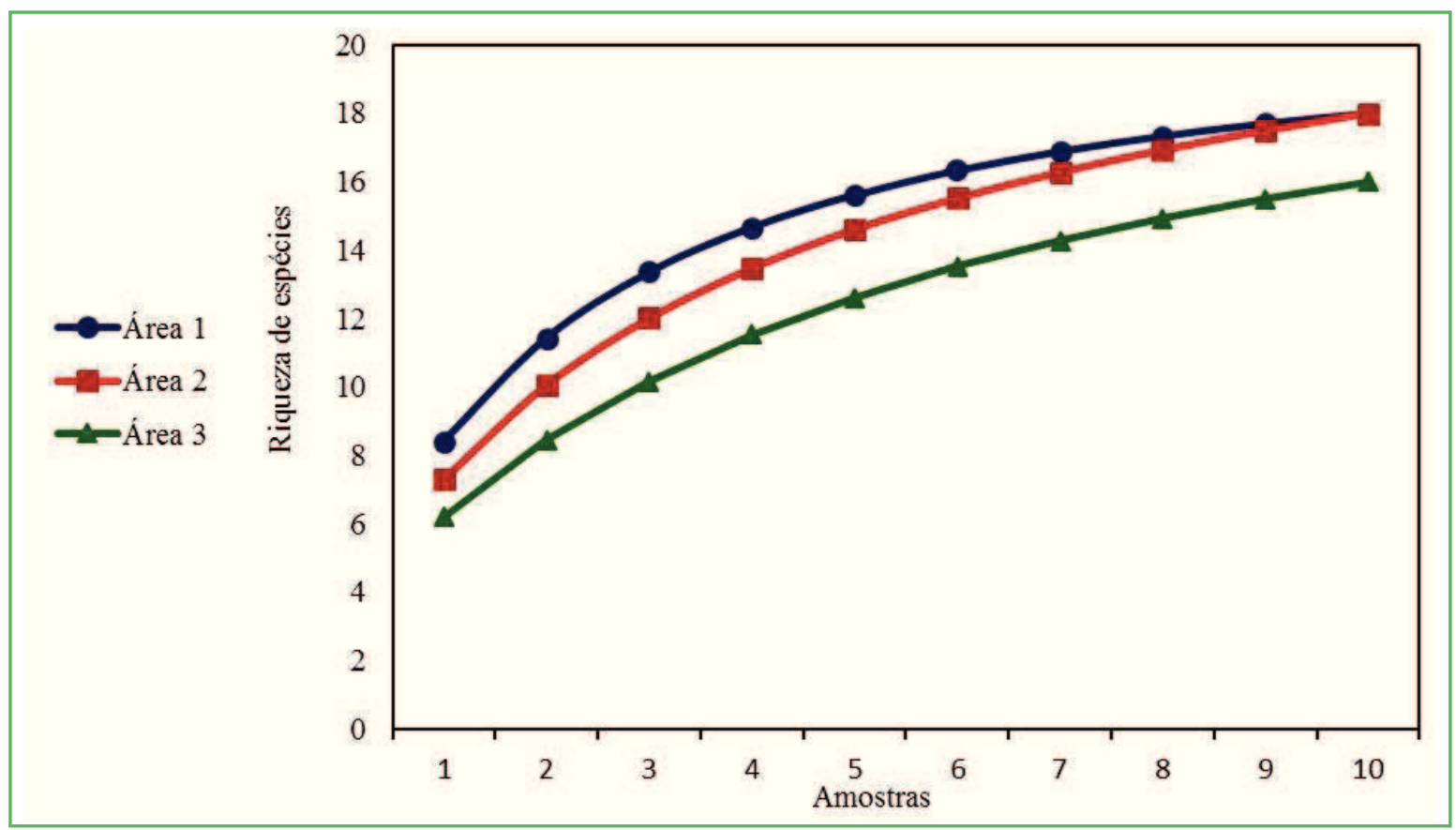


O dendrograma comparando a similaridade das assembleias mostra que os organismos encontrados em todas as áreas são bastante semelhantes (mais de $60 \%$ de similaridade), mas há formação de dois grupos (Figura 4), um grupo constituído pelas áreas de vegetação secundária e Área Antropizada e o outro grupo formado apenas pela assembleia da Área Conservada. Este resultado mostra que a fauna de Scarabaeinae da Área Conservada é um pouco diferente dos outros sítios, o que pode ser explicado pela maior abundância de indivíduos.

FIGURA 4: Similaridade entre as assembleias de Scarabaeinae em três áreas do Parque Estadual Vitório Piassa. Área Conservada (Área 1); Área Secundária (Área 2); Área Antropizada (Área 3).

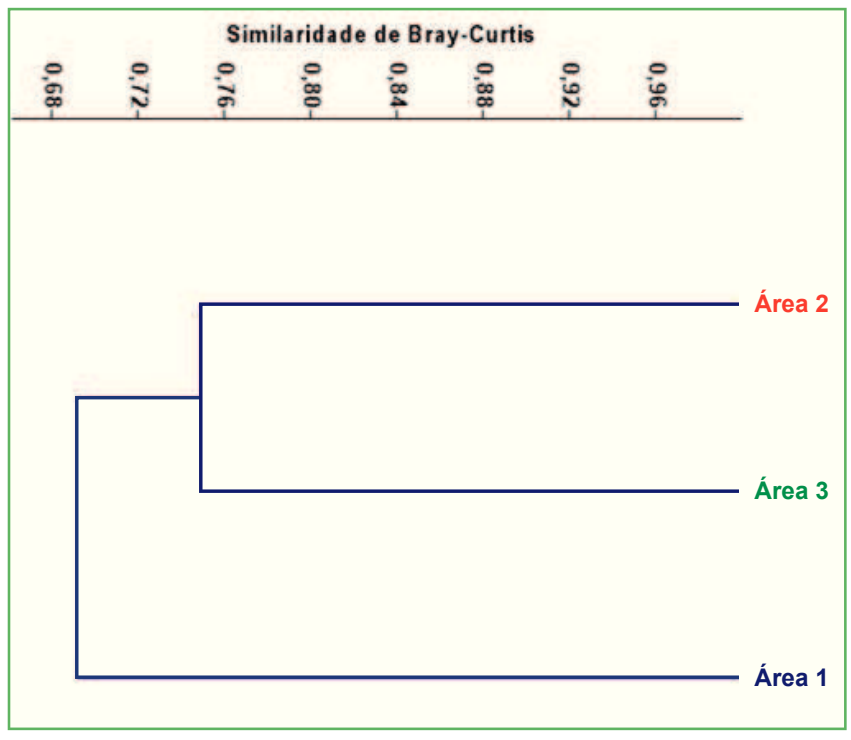

\section{Discussão}

Os besouros escarabeíneos coletados nos três sítios amostrados apresentaram 22 espécies no total, o que pode ser considerado uma grande quantidade de espécies ainda presentes, considerando-se o uso que a área do PEVP vem sofrendo ao longo dos anos. Além disso, foi possível observar que, na área mais antropizada do parque, foi encontrado um número menor de espécies, o que pode ser devido às alterações ambientais que afetaram diretamente a riqueza, assim como a abundância destes indivíduos (LEWINSOHN et al., 2005). As principais perturbações ambientais que remetem à perda de espécies nos ecossistemas são causadas principalmente por pressões antrópicas (WINK et al., 2005; BEGON et al., 2007; GARDNER et al., 2008), o que pode ser visto na Área Antropizada já que ela encontra-se com presença mínima de vegetação mais densa, consequentemente, uma faixa ao longo desse fragmento é exposta a altas temperaturas, causadas pelo aumento da luminosidade solar, e à constante incidência de ventos, que levam à derrubada de árvores que antes contribuíam para a conservação da umidade do solo. Como resultado, algumas espécies adaptadas aos fatores internos da floresta, como umidade e sombreamento, são eliminadas por não se adaptarem à nova condição imposta àquele habitat (LAURANCE; VASCONCELOS, 2009).

Alterações em relação à riqueza e abundância de diferentes espécies são encontradas em ambientes fragmentados, o que mostra a relação entre a mudança na composição de espécies e as fragmentações de habitat, causadas principalmente pelas ações antrópicas nos ambientes naturais (ALMEIDA; LOUZADA, 2009). Algumas espécies são bastante sensíveis aos efeitos da fragmentação de ambientes, como, por exemplo, os insetos, sapos, mamíferos e aves insetívoras (BIERREGAARD JR. et al., 1992). Os insetos, em especial, são fortemente influenciados pela cobertura do dossel e altura de serapilheira (HALFFTER; ARELLANO, 2002), e frequentemente são percebidos efeitos sobre a riqueza e a composição de espécies nesses ambientes fragmentados (PRIMACK; RODRIGUES, 2001; LAURANCE; VASCONCELOS, 2009). Halffter e Arellano (2002) analisaram os efeitos da fragmentação sobre a biodiversidade de escarabeíneos e demonstraram que, em áreas onde a cobertura florestal foi modificada, espécies nativas foram substituídas por espécies de áreas abertas. Em relação aos insetos coletados no PEVP, foi possível observar que os besouros encontrados nas áreas de coleta de vegetação secundária e Área Antropizada diferem levemente das espécies existentes na Área Conservada, com vegetação densa devido à maior abundância de indivíduos. $\mathrm{O}$ fato de a vegetação secundária apresentar um maior índice de diversidade pode ser explicado através da Hipótese do Distúrbio Intermediário (CONNELL, 1978), que afirma que áreas intermediárias podem conter uma alta diversidade, pois podem comportar espécies adaptadas a ambientes mais preservados e também espécies de áreas mais degradadas, 
ou seja, áreas de florestas e áreas mais abertas (SANTOS, 2004; DIAS et al., 2006; GIACOMINI, 2007; COSTA et al., 2011; FREY, 2011). Este sítio de amostragem se caracteriza por sua vegetação secundária e o fato de estar localizado em uma clareira no meio da mata, mostra que este sítio abriga espécies que toleram ambientes diferentes.

Levando em consideração os índices de similaridade, foi possível ver que a Área Conservada apresenta menor similaridade com as demais áreas, mostrando que áreas com vegetação mais densa abrigam maior abundância de indivíduos, o que pode refletir em maiores funções ecossistêmicas, como aeração do solo, decomposição e ciclagem de nutrientes e dispersão secundária de sementes (HALFFTER; FAVILA, 1993; LEWINSOHN et al., 2005; NICHOLS et al., 2009; OLIVEIRA et al., 2011). Portanto, os resultados apresentados através das coletas dos besouros indicadores no PEVP mostram que o fragmento ainda mantém uma alta diversidade de espécies e que áreas que sofrem maior pressão antrópica apresentam mudanças na composição e estrutura das suas assembleias.

\section{Agradecimentos}

A Alcides Abraão Titton Lisboa pelo auxílio nos trabalhos em campo. Ao professor Dr. Fernando Zagury Vaz-de-Mello (UFMT) pela identificação de algumas espécies. Ao Dr. Pedro Giovâni da Silva pela ajuda na identificação de espécies e auxílio na análise de dados. Ao Dr. Jorge Jamhour pela cessão do Laboratório de Entomologia, Zoologia e Apicultura (UTFPR).

\section{Referências}

ALMEIDA, S. S. P.; LOUZADA, J. N. C. Estrutura da comunidade de Scarabaeinae (Scarabaeidae: Coleoptera) em fitofisionomias do Cerrado e sua importância para a conservação. Neotropical Entomology, Piracicaba, v. 38, n. 1, p. 32-43, 2009.

AUDINO, L. D.; NOGUEIRA, J. M.; SILVA, P. G.; NESKE, M. Z.; RAMOS, A. H. B.; MORAES, L. P.; BORBA, M. F. S. Identificação dos coleópteros (Insecta: Coleoptera) das regiões de Palmas (município de Bagé) e Santa Barbinha (município de Caçapava do Sul), RS. Bagé: Embrapa (Documentos, 70), 2007. $92 \mathrm{p}$.

BEGON, M.; TOWNSEND, C. R.; HARPER, J. L. Ecologia: de indivíduos a ecossistemas. 4. ed. Porto Alegre: Artmed, 2007. 752 p.
BENSUSAN, N. Conservação da biodiversidade em áreas protegidas. Rio de Janeiro: Editora FGV, 2006. 225 p.

BIERREGAARD JR., R. O.; LOVEJOY, T. E.; KAPOS, V.; DOS SANTOS, A. A.; HUTCHINGS, R. W. The biological dynamics of tropical rainforest fragments. BioScience, Berkeley, v. 42, n. 11, p. 859-866, 1992.

BOGONI, J. A.; GRAIPEL, M. E.; CASTILHO, P. V.; FANTACINI, F. M.; KUHNEN, V. V.; LUIZ, M. R.; MACCARINI, T. B.; MARCON, C. B.; TEIXEIRA, C. S. P.; VAZ-DE-MELLO, F. Z; HERNÁNDEZ, M. I. M. Contributions of the mammal community, habitat structure, and spatial distance to dung beetle community structure. Biodiversity and Conservation, New York, v. 25, n. 9, p. 1661-1675, 2016.

BROWN, K. S. Insetos como rápidos e sensíveis indicadores de uso sustentável de recursos naturais. In: MARTOS, H. L.; MAIA, N. B. (Ed.). Indicadores ambientais. 1. ed. Sorocaba: s.n., 1997. p. 143-151.

CARVALHO, F. A.; NASCIMENTO, M. T.; OLIVEIRA, P. P.; RAMBALDI, D. M.; FERNANDES, R. V. A importância dos remanescentes florestais da Mata Atlântica de baixada costeira fluminense para a conservação da biodiversidade na APA da Bacia do Rio São João/Mico-Leão-Dourado - RJ. In: CONGRESSO BRASILEIRO DE UNIDADES DE CONSERVAÇÃO, IV, 2004, Curitiba. Anais... Curitiba: Fundação O Boticário de Proteção à Natureza, 2004. p. 106-113.

COLWELL, R. K. EstimateS: statistical estimation of species richness and shared species from samples. 2005. Disponível em: $<$ http://viceroy.eeb.uconn.edu/estimates $>$.

CONNELL, J. H. Diversity in tropical rainforest and coral reefs. Science, New York, v. 199, p. 1302-1310, 1978.

COSTA, M. do P.; PEREIRA, J. A. A.; FONTES, M. A. L.; DE MELO, P. H. A.; HENDRIGO, P.; SALGADO, D.; PELLICCIOTTII, A. S.; SILVA, R. A. Estrutura e diversidade da comunidade arbórea de uma floresta superomontana, no planalto de Poços de Caldas (MG). Ciência Florestal, Santa Maria, v. 21, n. 4, p. 711-725, 2011.

DIAS, A.; MARTINELLI, C.; VEIGA, L. G.; MATTOS, R. G.; ARANHA, T. P. Abertura de dossel e riqueza de espécies em um fragmento de cerrado de Itirapina, SP. 2006. Disponível em: <http://www2.ib.unicamp.br/profs/fsantos/ecocampo/2008/ Relatorios/Perturbacao.pdf $>$.

FREITAS, A. V. L.; LEAL, I. R.; UEHARA-PRADO, M.; IANNUZZI, L. Insetos como indicadores de conservação da paisagem. In: ROCHA, C. F. D.; BERGALLO, H. G.; VAN SLUYS, M.; ALVES, M. A. S. (Ed.). Biologia da conservação: essências. São Carlos: RiMa Editora, 2006. p. 357-384.

FREY, G. Variação na riqueza de espécies entre e dentro de zonas em ambiente de costão rochoso. 2011. Disponível em: $<$ http://ecologia.ib.usp.br/curso/2011/pdf/gabriel_frey.pdf $>$.

GARDNER, T. A.; HERNÁNDEZ, M. I. M.; BARLOW, J.; PERES, C. A. Understanding the biodiversity consequences of habitat change: the value of secondary and plantation forests for neotropical dung beetles. Journal of Applied Ecology, London, v. 45, p. 883-893, 2008.

GIACOMINI, H. C. Os mecanismos de coexistência de espécies como vistos pela teoria ecológica. Oecologia Brasiliensis, Rio de Janeiro, v. 11, n. 4, p. 21-543, 2007. 
HALFFTER, G. Historical and ecological factors determining the geographical distribution of beetles (Coleoptera: Scarabaeidae: Scarabaeinae). Folia Entomologica Mexicana, Xalapa, v. 82, p. 195-238, 1991.

HALFFTER, G.; ARELLANO, L. Response of dung beetle diversity to human-induced changes in a tropical landscape. Biotropica, Malden, v. 34, p. 144-154, 2002.

HALFFTER, G.; FAVILA. M. E. The Scarabaeinae (Insecta: Coleoptera) an animal group for analyzing, inventorying and monitoring biodiversity in tropical rainforest and modified landscapes. Biology International, Paris, v. 27, p. 15-21, 1993.

HAMMER, Ø.; HARPER, D. A. T.; RYAN, P. D. PAST: paleontological statistics software package for education and data analysis. 2001. Palaeontologia Electronica, PAST, 3.0. Disponível em: <http://palaeo-electronica.org/2001_1/past/past.pdf >.

KLEIN, B. C. Effects of forest fragmentation on dung and carrion beetle communities in Central Amazonia. Ecology, New York, v. 70, p. 1715-1725, 1989.

LAURANCE, W. F.; VASCONCELOS, H. L. Conseqüências ecológicas da fragmentação florestal na Amazônia. Oecologia Australis, Rio de Janeiro, v. 13, n. 3, p. 434-451, 2009.

LEWINSOHN, T. M.; FREITAS, A. V. L.; PRADO, P. I. Conservação de invertebrados terrestres e seus habitats no Brasil. Megadiversidade, Belo Horizonte, v. 1, n. 1, p. 62-69, 2005.

LOPES, J.; KORASAKI, V.; CATELLI, L. L.; MARÇAL, V. V. M.; NUNES, M. P. B. P. A comparison of dung beetle assemblage structure (Coleoptera: Scarabaeidae: Scarabaeinae) between an Atlantic forest fragment and adjacent abandoned pasture in Paraná, Brazil. Zoologia, Curitiba, v. 28, n. 1, p. 72-79, 2011.

MMA - MINISTÉRIO DO MEIO AMBIENTE. Unidades de Conservação. 2014. Disponível em: <http://www.mma.gov.br/ areas-protegidas/unidades-de-conservacao $>$.

MORSELLO, C. Áreas protegidas públicas e privadas: seleção e manejo. São Paulo: ANNABLUME, 2001. 343 p.

MYERS, N.; MITTTERMEYER, R. A.; MITTERMEYER, C. G.; FONSECA, A. B.; KENT, J. Biodiversity hot spots for conservation priorities. Nature, London, v. 403, n. 6772, p. 853-858, 2000.

NICHOLS, E.; GARDNER, T. A.; PERES C. A.; SPECTOR, S. Co-declining mammals and dung beetles: an impending ecological cascade. Oikos, Copenhagen, v. 118, p. 481-487, 2009.

OLIFIERS, N.; CERQUEIRA, R. Fragmentação de habitat: efeitos históricos e ecológicos. Biologia da conservação: (C.F.D. ROCHA; H.G. BERGALLO; M.V. SLUYS \& M. A. S. ALVES, (Org.). RiMa Editora: São Carlos, p. 261-279, 2006.

OLIVEIRA, V. H. F.; MOTA-SOUZA, J. G. M.; VAZ-DE-MELLO, F. Z.; de SIQUEIRA NEVES, F.; FAGUNDES, M. Variação na fauna de besouros rola-bosta (Coleoptera: Scarabaeinae) entre habitats de cerrado, mata seca e mata ciliar em uma região de transição cerrado-caatinga no norte de Minas Gerais. MG.BIOTA, Belo Horizonte, v. 4, n. 4, p. 4-16, 2011.

PAGLIA, A. P.; FERNANDEZ, F. A; DE MARCO JR., P. Efeitos da fragmentação de habitats: quantas espécies, quantas populações, quantos indivíduos, e serão eles suficientes. In: ROCHA, C. F. D.; BERGALlO, H. G.; VAN SLUYS, M.; ALVES, M. A. S. (Ed.). Biologia da conservação: essências. São Carlos: RiMa Editora, 2006. p. 281-316.
PIRES, A. S.; FERNANDEZ, F. A.; BARROS, C. S.; ROCHA, C. F. D.; BERGALLO, H. G. Vivendo em um mundo em pedaços: efeitos da fragmentação florestal sobre comunidades e populações animais. In: ROCHA, C. F. D.; BERGALLO, H. G.; VAN SLUYS, M.; ALVES, M. A. S. (Ed.). Biologia da conservação: essências. São Carlos: RiMa Editora, 2006. p. 231-260.

PRIMACK, R. B.; RODRIGUES, E. Biologia da conservação. Londrina: E. Rodrigues, 2001. 328 p.

RODERJAN, C. V.; GALVÃO, F.; KUNIYOSHI, Y. S.; HATSCHBACH, G. G. As unidades fitogeográficas do estado do Paraná, Brasil. Ciência \& Ambiente, Santa Maria, v. 24, p. 75-92, 2002.

SANTOS, B. A. Influência da distância geográfica na similaridade florística de clareiras e sub-bosques em uma floresta de terra firme na Amazônia Central. 2004. Disponível em: <http://pdbff.inpa.gov.br/cursos/efa/livro/2004/PDFs/41_final/ braulio.pdf $>$.

SILVA, R. J.; COLETTI, F.; COSTA, D. A.; VAZ-DE-MELLO, F. Z. Rola-bostas (Coleoptera: Scarabaeidae: Scarabaeinae) de florestas e pastagens no sudoeste da Amazônia brasileira: Levantamento de espécies e guildas alimentares. Acta Amazonica, Manaus, v. 44, n. 3, p. 345-352, 2014.

SOFIA, S. H.; SUZUKI, K. M. Comunidades de machos de abelhas Euglossina (Hymenoptera: Apidae) em fragmentos florestais no sul do Brasil. Neotropical Entomology, Piracicaba, v. 33, n. 6, p. 693 $702,2004$.

SNUC - SISTEMA NACIONAL DE UNIDADES DE CONSERVAÇÃO DA NATUREZA. Lei no 9.985, de 18 de julho de 2000. Brasília: MMA/SBF, 2000.

SNUC - SISTEMA NACIONAL DE UNIDADES DE CONSERVAÇÃO DA NATUREZA. Sistema Nacional de Unidades de Conservação da Natureza - SNUC, lei no 9.985, de 18 de julho de 2000; decreto no 4.340, de 22 de agosto de 2002 . 6. ed. Brasília: MMA/SBF, 2006. 56 p.

SOS MATA ATlÂNTICA. A Mata Atlântica. 2014. Disponível em: <http://www.sosma.org.br/nossa-causa/a-mata-atlantica/>.

TABARELli, M.; PINTO, L. P.; SILVA, J. M. C.; HIROTA, M. M.; BEDÊ, L. C. Desafios e oportunidades para a conservação da biodiversidade na Mata Atlântica brasileira. Megadiversidade, Belo Horizonte, v. 1, n. 1, p. 132-138, 2005.

THOMAZINI, M. J.; THOMAZINI, A. P. B. W. A fragmentação florestal e a diversidade de insetos nas florestas tropicais úmidas. Rio Branco: EMBRAPA Acre. (Documentos, 57). 2000. 21 p.

VOLTOLINI, S. Retorno 3: ciclo da madeira em Pato Branco. Pato Branco: Imprepel, 2000. 274 p.

WINK, C.; GUEDES, J. V. C.; FAGUNDES, C. K.; ROVEDDER, A. P. Insetos edáficos como indicadores da qualidade ambiental. Revista de Ciências Agroveterinárias, Lages, v. 4, n. 1, p. 60-71, 2005.

WWF BRASIL. Sistema Nacional de Unidades de Conservação (SNUC) completa 14 anos de avanços e desafios. 2014. Disponível em: <http://www.wwf.org.br/wwf_brasil/?40382/ Sistema-Nacional-de-Unidades-de-Conservacao-SNUC-completa14-anos-de-avancos-e-desafios>. 